\title{
Knockdown of hTERT by siRNA inhibits cervical cancer cell growth in vitro and in vivo
}

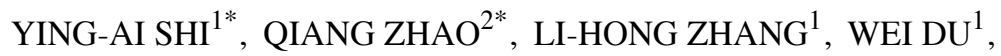 \\ XUE-YAO WANG ${ }^{3}, \mathrm{XU} \mathrm{HE}^{1}$, SHAN WU $^{1}$ and YU-LIN LI ${ }^{1}$ \\ ${ }^{1}$ Key Laboratory of Pathobiology, Ministry of Education, School of Basic Medical Sciences, \\ ${ }^{2}$ Department of Pediatric Surgery, The First Hospital, ${ }^{3}$ Norman Bethune College \\ of Medical Sciences, Jilin University, Changchun 130021, P.R. China
}

Received March 20, 2014; Accepted May 15, 2014

DOI: 10.3892/ijo.2014.2493

\begin{abstract}
Human telomerase reverse transcriptase (hTERT) is the catalytic component of telomerase that facilitates tumor cell invasion and proliferation. It has been reported that telomerase and hTERT are significantly upregulated in majority of cancers including cervical cancer, thus, downregulation of hTERT is a promising target in malignant tumor treatment. We established a short interfering RNA (siRNA) targeting hTERT, and transfected it into HeLa cells (a cervical cancer cell line) to investigate the effect of cell proliferation, apoptosis, migration and invasion in cervical cancer cells. The results showed that siRNA targeting hTERT could effectively knock down hTERT expression, remarkably suppress telomerase activity, cell proliferation, migration and invasion, and induced cell apoptosis of cervical cancers cells in vitro. In addition, we evaluated whether siRNA targeting hTERT affects tumor growth in nude mice, and found that it dramatically inhibited tumorigenesis and growth of mice injected with siRNA targeting hTERT. Furthermore, we also found that knockdown of hTERT was able to significantly suppress constitutive phosphorylation of Akt, PI3K, which might imply that reduction of hTERT inhibited tumor growth via the PI3K/ Akt signaling pathway to some extent. These results suggest that the suppression of hTERT expression by siRNA inhibits cervical cancer cell growth in vitro and in vivo, and may provide a novel target for anticancer gene therapy.
\end{abstract}

\section{Introduction}

Cervical cancer is caused by a multistep process that involves transformation of the normal cervical epithelium to a preneo-

Correspondence to: Professor Yu-Lin Li, Key Laboratory of Pathobiology, Ministry of Education, School of Basic Medical Sciences, Jilin University, Changchun 130021, P.R. China

E-mail: liyulin318@sina.com

${ }^{*}$ Contributed equally

Key words: cervical cancer, human telomerase reverse transcriptase, tumor growth, short interfering RNA plastic cervical intraepithelial neoplasia that is subsequently transformed to invasive cervical cancer $(1,2)$. The incidence and mortality of invasive cervical cancer have steadily decreased (3), and cervical cancer remains the third most common cancer in women worldwide (4) and the leading malignancy in developing countries, accounting for $83 \%$ of all cancer cases (5). Although well organized screening and early therapeutic schedules have been carried out, the occurrence of invasive cervical cancer remains high in developing areas (6). Furthermore, as the understanding of key cellular pathways involved in tumor growth has improved, molecular targeted therapies have been widely exploited. Therefore, the development of new therapeutic strategies bases on molecular targeted therapies is necessary to improve survival in patients with cervical cancer.

Telomerase plays a key role in conferring immortality to cancer cells through regulation of telomere length $(7,8)$. It synthesizes the telomeric repeats at the ends of chromosomes and replaces the progressively lost end sequences during each cell cycle, allowing cells to escape mortality and continue to proliferate. The reverse transcriptase telomerase is composed of two core components: a ubiquitously expressed RNA component (hTR), and a catalytic subunit human telomerase reverse transcriptase (hTERT) which expression is limited to the formation of a catalytically active enzyme (9) and regulating telomerase activity (10-12). Increased telomerase activity (TA) is found in $90 \%$ of human cancer cells $(13,14)$, yet, telomerase activity is at low level or undetectable in most normal human somatic cells. Therefore, inhibition of hTERT could be an effective antitumor strategy.

Growth evidence has demonstrated that inhibiting telomerase; especially hTERT, by genetic, antisense RNAi is a highly promising for cancer therapy as already demonstrated in several cancer cell lines (15-18). Zhang et al transfected a plasmid encoding hTERT-specific shRNAs into human hepatocellular carcinoma cell lines and found that they could stably suppress hTERT expression, which led to the inhibition of cell proliferation and to an attenuated tumorigenic potency (19). Dong et al showed that transfection of encoding hTERT-specific siRNAs into human breast cancer cell lines could inhibit cell proliferation and induced cell apoptosis (20). Recently, several studies also demonstrated that the 
knockdown of the hTERT via siRNA effectively inhibited the expression of telomerase activity and cell proliferation, and the cell cycle arrest of cervical cancer cells in vitro (17,21-24). However, these studies mainly focus on effect of silencing hTERT on cell proliferation and cell apoptosis in vitro, little attention has been given to reduction hTERT affect on tumor growth of cervical cancer in vivo. In the present study, we examined the effect of hTERT knockdown by siRNA on cell proliferation, cell apoptosis, cell migration and invasion in a human cervical cancer cell line (HeLa cells) in vitro and on tumor growth in cervical cancer xenografts in vivo.

\section{Materials and methods}

Cell culture. The human cervical carcinoma cell lines, HeLa cells were purchased from Cell Bank of Type Culture Collection of Chinese Academy of Sciences, Shanghai Institute of Cell Biology, Chinese Academy of Sciences (Shanghai, China). HeLa cells were cultured in RPMI-1640 medium (Invitrogen, Carlsbad, CA, USA) supplemented with heat-inactivated $10 \%$ fetal bovine serum (FBS) (Biochrom AG) and $1 \%$ penicillin/ streptomycin at $37^{\circ} \mathrm{C}$ in a humidified atmosphere containing $5 \%$ $\mathrm{CO}_{2}$.

Design and transfection of short interfering RNA. siRNAs were designed to target different regions of the coding sequences of the hTERT mRNA (GenBank accession no. AF015950) according to Reynolds et al (25). Selected sequences were submitted to blast search in the GenBank database to confirm that only the hTERT gene was targeted. Short hairpin RNA (shRNA) targeting the TLR4 transcript was synthesized and annealed. The synthesized oligonucleotides contain specific target sequence, a loop, the reverse complement of the target sequence, a stop codon for U6 promoter and two sticky ends. The target sequences in the oligonucleotide for suppressing hTERT: siRNAsequence: GTCTGCCGTTGCCCAAGAG (sense); Sequences for the scrambled siRNA: AATTCTCCGAACGTGTCACGT (sense), which does not target any gene product and have no significant sequence similarity to human gene sequences, and was used as control to determine the effects of siRNA delivery. The siRNA and scramble sequence were cloned into expressing plasmid pGCsilencer (Genechem, Shanghai, China), respectivly, and transiently transfected into HeLa cells using Lipofectamine ${ }^{\mathrm{TM}} 2000$ reagent (Invitrogen) according to the manufacturer's instructions. Transfection efficiency was evaluated by a fluorescence microscope. Transfection was screen by G418 (Invitrogen) and obtained from two siRNA clones and one scramble clone, named as T1, T2 and N, respectively. HeLa cells without transfection was used as parental control.

Real-time PCR. T1, T2, N and HeLa cells were harvested for RNA extraction following culture for $72 \mathrm{~h}$. RNA was insolated using TRIzol reagent (Invitrogen). RNA was reverse-transcribed into cDNA by a Primescript ${ }^{\mathrm{TM}}$ RT reagent kit based on the manufacturer's protocols (Takara, Dalian, China). Quantitative real-time polymerase chain reaction (RT-PCR) assays were carried out using SYBR-Green Real-Time PCR Master Mix (Toyobo, Osaka, Japan) and
RT-PCR amplification equipment using specific primers: forward primers, 5'-GGAGCAAGTTGCAAAGCATTG-3' and reverse, 5'-TCCCACGACGTAGTACATGTT-3'; GAPDH forward primers, 5'-TGTGGGCATCAATGGATTTGG-3' and reverse, 5'-ACACCATGTATTCCGGGTCAAT-3'. The PCR conditions were: a pre-denaturing at $95^{\circ} \mathrm{C}$ for $5 \mathrm{~min}$, followed by 40 cycles of denaturation at $95^{\circ} \mathrm{C}$ for $10 \mathrm{sec}$, annealing/ extension at $54^{\circ} \mathrm{C}$ for $20 \mathrm{sec}$, final extention $72^{\circ} \mathrm{C}$ for $5 \mathrm{~min}$. The amplification specificity was checked by melting curve analysis. Quantitative data were analyzed by using the Light Cycler software version 3.5 (Roche, Mannheim, Germany) and relative quantification of hTERT mRNA was derived by the $2^{-\Delta \Delta \mathrm{CT}}$ method, as previous described (26).

Western blot analysis. Cells were dissociated with trypsin (Gibco) and collected into $1.5 \mathrm{ml}$ EP tubes. The cells were washed twice with prechilled PBS ( $\mathrm{pH}$ 7.2) after centrifugation and were then lysed on ice for $30 \mathrm{~min}$ in $60 \mu \mathrm{l}$ cell lysis buffer ( $1 \mathrm{ml}$ RIPA + $10 \mu$ l PMSF, Beyotime). The cell lysates were centrifuged at $4^{\circ} \mathrm{C}$, at $12,000 \mathrm{rpm}$ for $5 \mathrm{~min}$, and the supernatants were collected, and protein concentrations were determined using the Bradford reagent (Sigma). Lysates were separated on 8 or $15 \%$ SDS-PAGE; proteins were transferred to Immobilon membrane (Millipore, Bedford, MA) immunoblotted with specific primary antibodies and incubated with corresponding horseradish peroxidase-conjugated secondary antibody. Protein bands were visualized with enhanced chemiluminescence reagent (ECL, Amersham, GE Healthcare, Velizy-Villacoublay, France). The primary antibodies used in the western blots were: antibodies against TLR4, $\beta$-actin, BCL-2 and survivin (Santa Cruz Biotechnology, Santa Cruz, USA); Akt, phosphorylated(p-) Akt, PI3K, p-PI3K, mTOR and p-mTOR (Sigma-Aldrich, St. Louis, MO, USA); Secondary Abs used for immunodetection were: HRP-conjugated goat anti-mouse IgG (Santa Cruz Biotechnology). A gel image analysis system was used to scan the membrane and analyze the intensity of each band.

Telomerase activity assay. Telomerase activity was determined with the conventional telomeric repeat amplification protocol (TRAP) using the TRAP Telo TAGGG PCR enzyme-linked immunosorbent assay (ELISA) kit (Roche) according to the manufacturer's protocol (27).

Southern blot analysis of telomere length. Genomic DNA of the cultured cells was isolated by the high pure template preparation kit (Roche) and telomere length was estimated by using the Telo TAGGG Telomere Length Assay kit (Roche). In brief, $2 \mu \mathrm{g}$ of genomic DNA was digested with restriction enzymes HinfI and RasI at $37^{\circ} \mathrm{C}$ for $2 \mathrm{~h}$ and separated on $0.8 \%(\mathrm{w} / \mathrm{v})$ gel. The DNA fragments were then transferred to a positively charged nylon membrane in $20 \mathrm{X}$ saline-sodium citrate buffer overnight at room temperature. The membrane was hybridized with a DIG-labeled telomere-specific probe and detected by an anti-DIG alkaline phosphatase and CDP Star as the chemiluminescence substrate. Telomere length was calculated using the Kodak Digital Sciences 1D ${ }^{\mathrm{TM}}$ sofeware (Roche).

Cell proliferation assay. To measure the effect of downregulation of hTERT by siRNA on cell proliferation, CCK-8 assay 
(Cell Counting Kit-8, Dojindo, Japan) was performed. In brief, $5 \times 10^{3}$ of cells were seeded into each well of a 96-well plate. The proliferative activity was determined at the end of different experimental periods $(24,48,72,96$ and $120 \mathrm{~h}$ ) using CCK-8 assay according to the manufacturer's instructions. In brief, $10 \mu 1$ of CCK- 8 was added to each well followed by incubation for an additional $2 \mathrm{~h}$. When the media changed from red to yellow, the absorbance value at a wavelength of $450 \mathrm{~nm}$ was detected by an enzymelinked immunosorbent assay reader (Thermo Labsystems, Finland). The experiment was performed at least three times with similar results.

The proliferation rate of cells was determined by measuring the incorporation of bromodeoxyuridine (BrdU) into the genomic DNA. In brief, $5 \times 10^{3}$ of cells were seeded into each well of a 96-well plate and cultured for $3 \mathrm{~h}$, and cells were treated with $20 \mu \mathrm{M}$ 5-bromo-2-deoxyuridine (BrdU, Sigma, Dallas, TX). After a 2-h incubation with BrdU, cells was fixed for $15 \mathrm{~min}$ in phosphate-buffered saline containing $4 \%$ paraformaldehyde. Cells were incubated with anti-BrdU IgGs (1:1000 dilution; Sigma) overnight at $4^{\circ} \mathrm{C}$, then washed with PBS containing $0.1 \%$ Triton X-100, and incubated for $1 \mathrm{~h}$ at $25^{\circ} \mathrm{C}$ in blocking buffer containing Cy3-conjugated anti-mouse IgGs (1:2000 dilution; Jackson Immunoresearch, West Grove, PA). Cells were washed three times, and counterstained with 4,6-diamidino-2-phenylindole (DAPI, $1 \mu \mathrm{g} / \mathrm{ml}$; Sigma). Coverslips were mounted in antifade ( $90 \%$ glycerol, $10 \% 1 \mathrm{M}$ Tris $\mathrm{pH} 8.0,0.2 \%$ propyl gallate) on glass slides and labeled cells were viewed by indirect immunofluorescence and ultraviolet microscopy. BrdU-labeled cells were counted from digital images taken from random fields for a total of 300 cells per coverslip.

Cell cycle analysis. Cells were harvested and washed with cold PBS, and then fixed with $75 \%$ ethanol at $-20^{\circ} \mathrm{C}$ overnight. The fixed cells were washed with cold PBS twice, then adding $500 \mu \mathrm{l}$ of DNA staining solution (containing $200 \mu \mathrm{g} /$ $\mathrm{ml} \mathrm{RNase} \mathrm{A}$ and $20 \mu \mathrm{g} / \mathrm{ml}$ propidium iodide staining solution (Whitehouse Station, NJ, USA) and incubated for $30 \mathrm{~min}$. Finally, the distribution of cells in the cell-cycle phases were analyzed from the DNA histogram with a FACS Caliber flow cytometer (Becton-Dickinson, San Jose, CA, USA) and CellQuest software (CA, USA).

Cell apoptosis assay. The percentage of apoptotic cells was assessed by the TUNEL technique following the manufacturer's instructions (In situ cell death detection kit, POD, Roche Diagnostic, Branchburg, NJ, USA). The number of apoptotic bodies were counted and averaged from three visual fields. In addition, we also detected caspase- 3 and caspase- 8 activity by ELISA as an additional indicator of apoptosis.

Caspase activity assay. The activity of caspase- 3 and caspase-8 was measured using caspase colorimetric protease assay kits (Millipore Corp., Billerica, MA, USA) according to the manufacturer's instructions. Briefly, cells were cultured for $24 \mathrm{~h}$, then washed twice with ice-cold PBS and harvested by centrifugation at $700 \mathrm{~g}$ for $10 \mathrm{~min}$. The cell pellets were then lysed in $150 \mu \mathrm{l}$ buffer provided in the kit. Protein concentrations of lysates were determined using the Lowry method
(28). Then, an aliquot of lysates $(80 \mu \mathrm{l})$ was incubated with $10 \mu \mathrm{l}$ substrate of each caspase at $37^{\circ} \mathrm{C}$ for $2 \mathrm{~h}$. Samples were analyzed at $405 \mathrm{~nm}$ using a microplate reader (Thermo Fisher Scientific, Inc., Waltham, MA, USA).

Migration assay. To assess the effect of downregulation of hTERT on cell migration, wound-healing assay was performed. In brief, the transfected cell lines were seeded on a 24-well plate and allowed to reach confluence. After scratching the bottom of the well with a pipette tip, the monolayer of cells was washed three times with PBS, and incubated in RPMI-1640 medium containing 1\% FBS for $24 \mathrm{~h}$; this medium was then replaced with RPMI-1640 medium containing $10 \%$ FBS. After $48 \mathrm{~h}$, cell migration was evaluated using an inverted phase-contrast microscope (Leica DMR, Germany).

Invasion assay. The invasiveness of silencing the downregulated hTERT by siRNA in vitro was measured using BD BioCoat ${ }^{\mathrm{TM}}$ Matrigel invasion chambers (Becton-Dickinson Labware, Bedford, MA, USA) according to the manufacturer's instructions. Filters were precoated on the upper side with Matrigel (1 mg/ml; BD Biosciences, San Jose, CA). The lower chamber was filled with culture media containing $10 \%$ FBS. Cells $\left(5 \times 10^{4}\right)$ were seeded in serum-free media in the upper chambers at $37^{\circ} \mathrm{C}$ for $24 \mathrm{~h}$. After incubation, cells invading the bottom surface of the filter were fixed and stained with $0.1 \%$ crystal violet in $20 \%$ methanol. Invasiveness was determined by counting the penetrating cells under a Nikon phase-contrast microscope and counted in $>10$ fields of view at x200 magnification.

Tumor growth in vivo. To investigate the effects of silencing the targeting hTERT on the tumorigenicity of xenografts and the influence on survival of tumor-burdened animals, 40 female BALB/nude mice (aged 4-6 weeks) were obtained from Tonghua Laboratory Animal Center (Beijing, China) and housed within a dedicated SPF facility at Laboratory Animal Center of Jilin University. T1, T2, N and HeLa $\left(1 \times 10^{8}\right)$ cells were subcutaneously injected into the right flank of mice, respectively. Tumor volume was measured by calipers every 5 days until mice were sacrificed under anesthesia. Each tumor was excised and weighed when mice were sacrificed on Day 21. Parts of each tumor tissue were wax embedded for H\&E stained to study cell apoptosis in vivo by TUNEL. All animal experiments were performed in accordance with institutional guidelines, following a protocol approved by the Ethics Committees of the Disease Model Research Center, Jilin University (Changchun, China).

Statistical analysis. All experiments were performed in triplicate and the data were recorded as mean \pm SD. Statistical comparison of more than two groups was performed using one-way ANOVA followed by the Tukey post-hoc test. Statistical analyses were undertaken using the SPSS ${ }^{\circledast}$ statistical package, version 19.0 (SPSS, Inc., Chicago, IL, USA) and the GraphPad Prism version 5.01 (GraphPad Software, San Diego, CA, USA) for Windows ${ }^{\circledR}$. P-values $<0.05$ were considered to be statistically significant. 

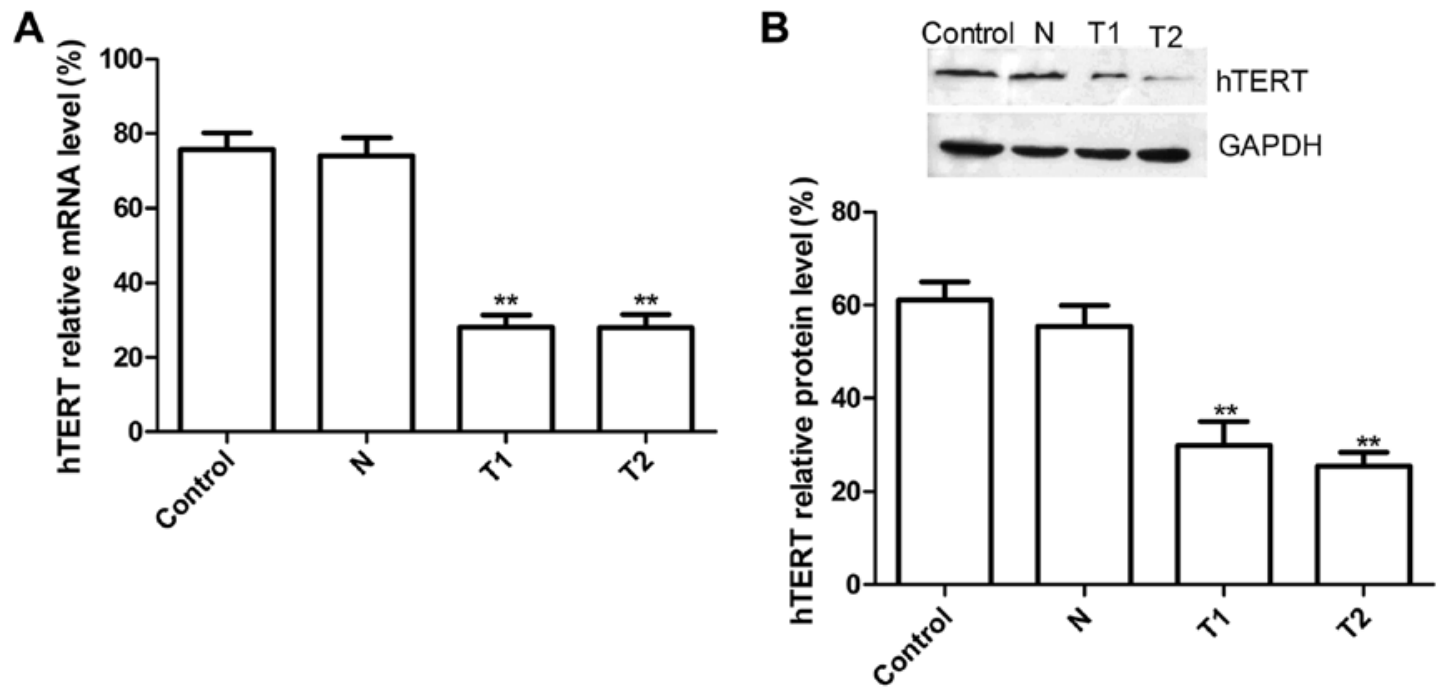

Figure 1. Knockdown of hTERT inhibits the hTERT expression in HeLa cancer cells. (A) Quantitative real-time PCR analysis of hTERT mRNA level (B) western blot analysis of hTERT protein level; T1 and T2, hTERT siRNA clone, N, scramble siRNA clone; Control, parental cells (untreated HeLa cell); ${ }^{* *} \mathrm{P}<0.01$ vs. control.

\section{Results}

Downregulation of hTERT $m R N A$ and protein by hTERT siRNA transfection. We designed sticky siRNAs cloned into the pGC silencer and and confirmed by sequencing that the recombinant plasmid construct met the requirements. The recombinant plasmids transfected into HeLa cells, were screed by G418 obtained from T1 and T2, two stable siRNA-hTERT clone cells, and then we tested their silencing efficiency both at the mRNA and protein levels in HeLa cells. Real-time RT-PCR results showed that hTERT mRNA expression in $\mathrm{T} 1$ and $\mathrm{T} 2$ group were significantly decreased compared to $\mathrm{N}$ group (scramble group) and control group (without transfection group) (Fig. 1A, P<0.01). There was no significant difference in $\mathrm{N}$ group and the control group; on protein level, there was no significant inhibition in hTERT protein expression in $\mathrm{N}$ group or the control group ( $\mathrm{P}>0.05)$, while the band density decreased dramatically in the $\mathrm{T} 1$ and $\mathrm{T} 2$ groups as compared with the $\mathrm{N}$ and the control group $(\mathrm{P}<0.01)$ (Fig. 1B). These results demonstrated that silencing hTERT was able to significantly decrease hTERT expression in cervical cancer cells $(\mathrm{P}<0.01)$.

Effect of hTERT siRNA treatment on telomere activity and length. Telomerase activity was detected by TRAP-PCR kit. The value of $\mathrm{T} 1(1.078 \pm 0.284)$ and $\mathrm{T} 2(1.030 \pm 0.218)$ were significantly decreased compared N $(2.806 \pm 0.477)$ and the parental cells $(2.810 \pm 0.348)$. The ratio of inhibition of telomerase activity in HeLa cells exposed to the $\mathrm{T} 1$ and $\mathrm{T} 2$ were both over $64.4 \%(\mathrm{P}<0.05)$, showing that downregulation could inhibit telomerase activity.

We then examined the effects of suppression of hTERT on telomere length. Analysis of terminal restriction fragments (TRFs) by Southern blotting demonstrated that the telomere lengths observed in $\mathrm{N}$ clones were $5-6 \mathrm{~kb}$, similar to that of parental cells. In contrast, telomere lengths in clones $\mathrm{T} 1$ and T2 showed significant shortening, with lengths averaging

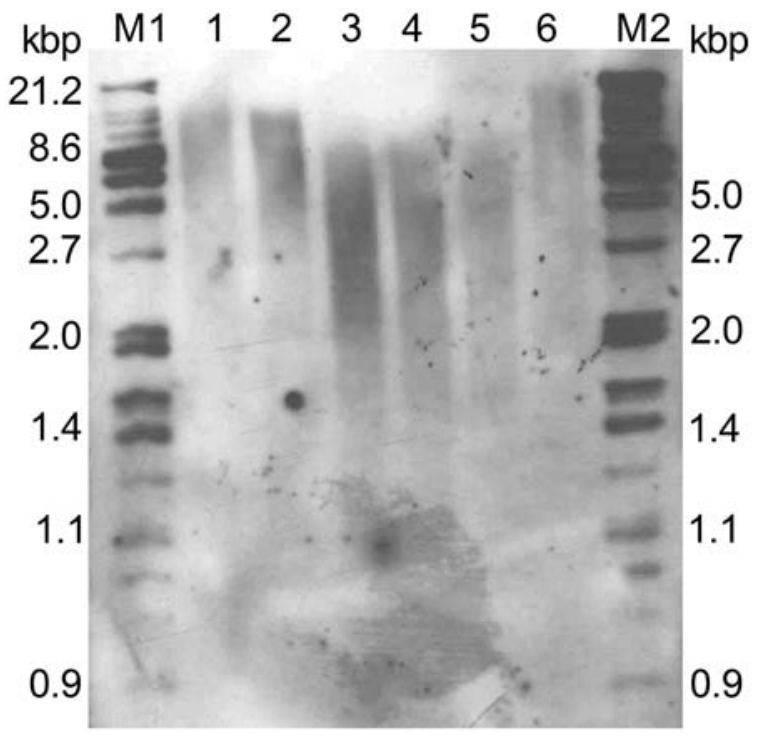

Figure 2. Southern blot showing the effects of hTERT siRNA on telomere length. M1 and M2, Marker; Lane 1, parental cells; Lane 2, scramble siRNA clone (N); Lane 3, hTERT siRNA clone (T1); Lane 4, hTERT siRNA clone (T2); Lane 5, control-DNA (3.4 kbp); Lane 6, control-DNA (10.2 kbp).

2-3 kb (Fig. 2). Taken together, these observations indicate that stable suppression of hTERT by RNAi functionally inhibits telomerase activity and length in human cervical cancer cells.

Effect of hTERT SiRNA on cell proliferation and the cell cycle. We examined the effects of silencing hTERT on tumor cervical cell growth in vitro. The anti-proliferative effect of silencing hTERT on HeLa tumor cells was examined using CCK-8 assays. The results clearly show that $\mathrm{T} 1$ and $\mathrm{T} 2$ clone cells significantly inhibited cell proliferation compared to $\mathrm{N}$ clone cell and parental cells at different time periods $(\mathrm{P}<0.01$, Fig. 3A). The proliferation rate of HeLa cells was determined using BrdU assay. As shown Fig. 3B, the proliferation rate of 

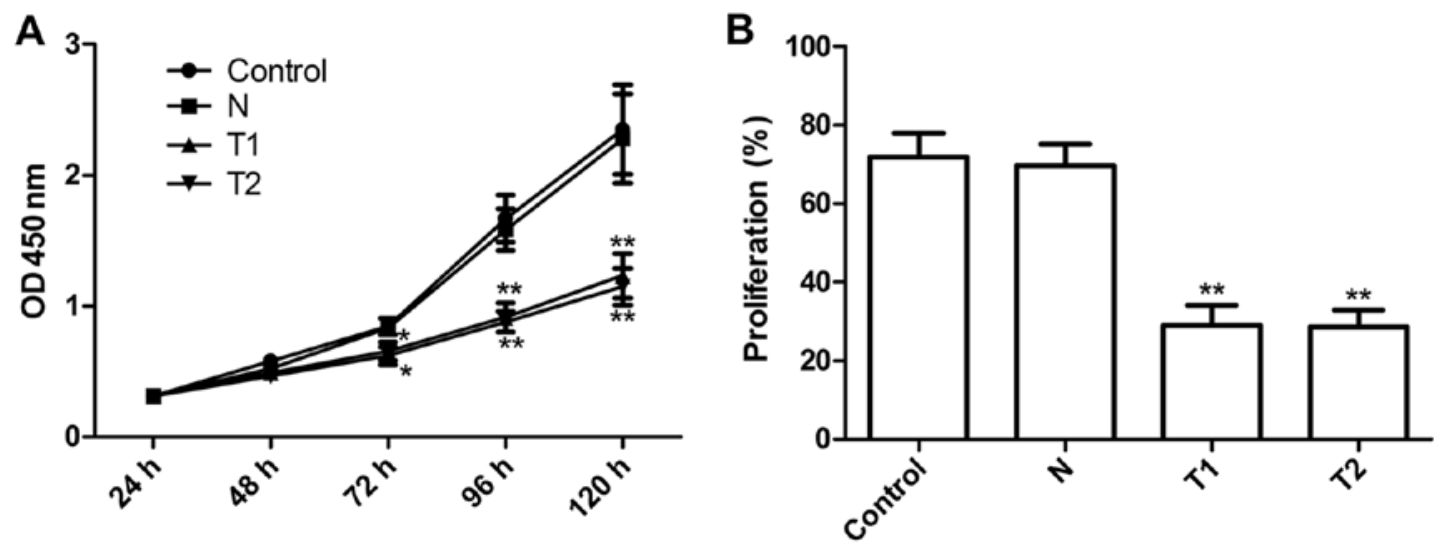

Figure 3. Knockdown of hTERT inhibits cancer cell proliferation. Cell proliferation was determined by CCK-8 assay (A) and BrdU assay (B); T1 and T2, hTERT siRNA clone; $\mathrm{N}$, scramble siRNA clone; Control, parental cell; ${ }^{*} \mathrm{P}<0.05$ and ${ }^{* * *} \mathrm{P}<0.01$ vs. control.

Table I. Cell cycle was determined by flow cytometry in different groups.

\begin{tabular}{lccrcc}
\hline Group & S & G0/G1 & G2/M & SPF (\%) & PI (\%) \\
\hline HeLa & 66.61 & 14.81 & 18.57 & 66.61 & 85.18 \\
N1 & 50.38 & 12.91 & 36.71 & 50.38 & 87.09 \\
T1 & $41.68^{\mathrm{a}}$ & $58.32^{\mathrm{b}}$ & 0.00 & $41.68^{\mathrm{a}}$ & $40.59^{\mathrm{a}}$ \\
T2 & $28.12^{\mathrm{b}}$ & $71.88^{\mathrm{b}}$ & 0.00 & $28.12^{\mathrm{a}}$ & $38.42^{\mathrm{a}}$ \\
\hline
\end{tabular}

T1 and T2, hTERT siRNA clone; N, scramble siRNA clone; Control, parental cell (untreated HeLa cell); ${ }^{\mathrm{a}} \mathrm{P}<0.05,{ }^{\mathrm{b}} \mathrm{P}<0.01$ vs. control.

$\mathrm{T} 1$ and $\mathrm{T} 2$ were significantly reduced the $\mathrm{N}$ and parental cells $(\mathrm{P}<0.01)$, in agreement with the CCK-8 assays. These results showed that silencing hTERT inhibits cell proliferation.

The effects of silencing hTERT on the cell cycles of HeLa cells were then analyzed by flow cytometry. The result showed that $\mathrm{T} 1$ and $\mathrm{T} 2$ had an increased percentage of arrest at the G0/G1 phase and a decreased percentage of arrest at the $\mathrm{S}$ phase compared with the $\mathrm{N}$ and parental cells $(\mathrm{P}<0.05$, Table I). In addition, SPF and PI were also reduced in $\mathrm{T} 1$ and $\mathrm{T} 2$ clone cells compared with the $\mathrm{N}$ and parental cells.

To investigate whether silencing hTERT could induce apoptosis, we analyzed apoptosis after treatment with hTERT siRNA. As shown Fig. 4A, T1 and T2 clone cells have substantial brown staining in the cell nucleus, whereas, the $\mathrm{N}$ clone and parental cells have a small amount of brown staining in the cell nucleus. Statistical analysis showed that the cell apoptosis ratio of $\mathrm{T} 1$ and $\mathrm{T} 2$ were significantly higher than those of the $\mathrm{N}$ clone and parental cells $(\mathrm{P}<0.01$, Fig. 4B).

To explore the possible mechanism of induction of cell apoptosis of silenced hTERT, caspase-3, caspase- 8 and caspase-10 activity was determined by ELISA. The results showed that caspase- 3 and caspase- 8 activity significantly increase in T1and $\mathrm{T} 2$ clone cells compared to the $\mathrm{N}$ clone and parental cell $(\mathrm{P}<0.01)$ (Fig. 4C and $\mathrm{D})$. Thus, silencing hTERT induced cell apoptosis of human cervical cancer cells.
Effect of hTERT siRNA on cell migration and invasion. To ascertain the inhibitory effect of silencing hTERT on cervical cancer migration, a wound-healing assay was performed. After 24-h of treatment, cells in the parental and the $\mathrm{N}$ clone cells efficiently spread into the wound area to such an extent that the wound boundary was not apparent, while only some cells of $\mathrm{T} 1$ and $\mathrm{T} 2$ clones spread forward in HeLa cells (Fig. 5A). Statistical analysis showed that the cell migration ratio of T1 and $\mathrm{T} 2$ was significantly reduced compared to the $\mathrm{N}$ clone and parental cells $(\mathrm{P}<0.01$, Fig. 5B).

The ability of the silenced hTERT to reduce the invasiveness of HeLa cells was then investigated by the transwell system. It was found that invasion was decreased significantly in $\mathrm{T} 1$ and $\mathrm{T} 2$ clone cells compared to the $\mathrm{N}$ clone and parental cells $(\mathrm{P}<0.01$, Fig. $5 \mathrm{C}$ and $\mathrm{D})$.

Effect of hTERT siRNA on tumor growth in a murine xenograft model. We investigated the effect of the silenced hTERT on tumor growth in nude mice with cervical cancer xenografts. At three days after the end of treatment, mice were sacrificed, and tumor weights were measured. As shown Fig. 6A, tumor weight was significantly less in the $\mathrm{T} 1$ and $\mathrm{T} 2$ clone cells than those of the $\mathrm{N}$ clone and parental cells. Tumor volume of all groups was measured, and the tumor volume was significantly lower in T1 and T2 clone cells on Days 10, 15 and 20 (Fig. 6B, $\mathrm{P}<0.01$ for all).

In addition, we determined tumor tissue cell apoptosis in vivo by TUNEL. The cell apoptosis ratio of T1 and T2 in vivo was significantly higher than those of the $\mathrm{N}$ clone and parental cells $(\mathrm{P}<0.01$, Fig. $6 \mathrm{C}$ and $\mathrm{D})$. These data demonstrated that the silencing of hTERT suppressed tumor growth of cervical cancer in vivo.

Effect of hTERT siRNA on the PI3K/AKT pathway. To clarify the molecular mechanisms involved in the silencing of hTERT inhibition of HeLa cell proliferation, we mainly focused on the effects of silencing hTERT on the activation of the PI3K/Akt pathway, which participate in the main intracellular signaling required for cell proliferation and survival. Our results demonstrated that silencing of hTERT inhibited the tyrosine phosphorylation of AKT and PI3K (Fig. 7). These 
A

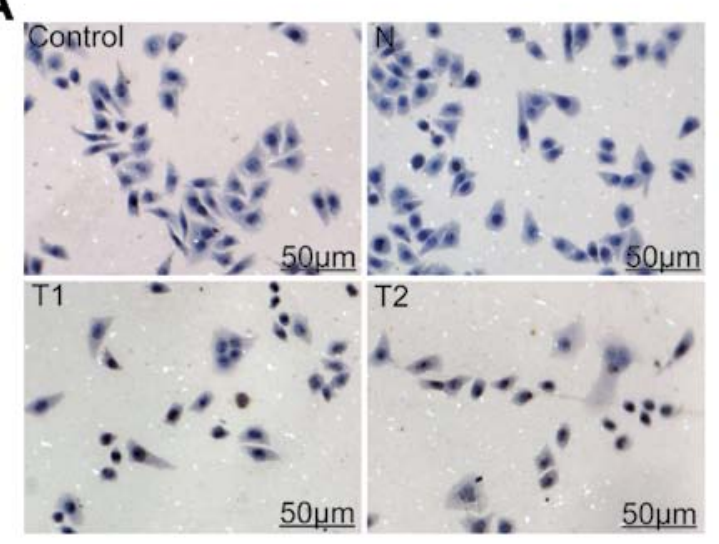

C

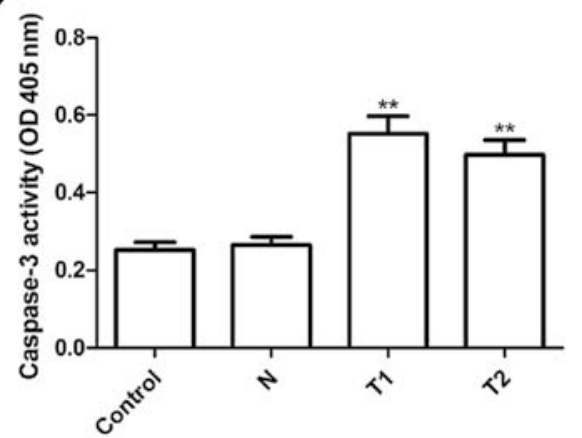

B

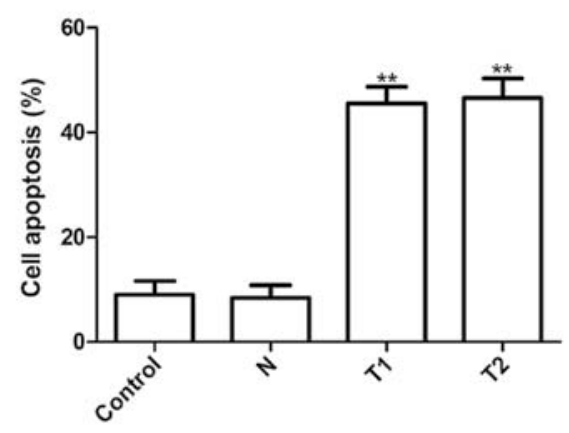

D

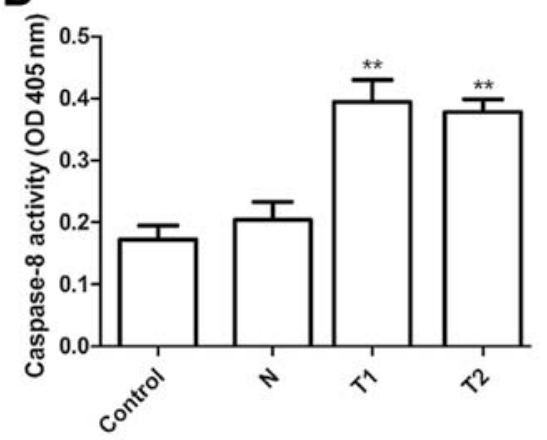

Figure 4. Knockdown of hTERT inhibits cancer cell apoptosis. Cell apoptosis was determined by TUNEL assay (A) and (B); Caspase-3 (C) and caspase-8 (D) activity of HeLa cells was measured; T1 and T2, hTERT siRNA clone; N, scramble siRNA clone; Control, parental cells; ${ }^{*} \mathrm{P}<0.05,{ }^{* *} \mathrm{P}<0.01$ vs. control.

\section{A}

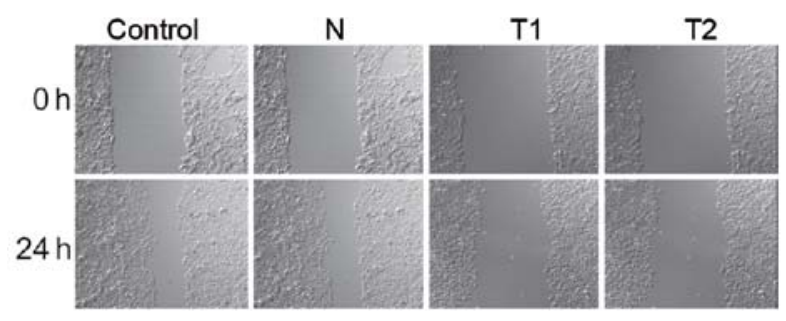

C
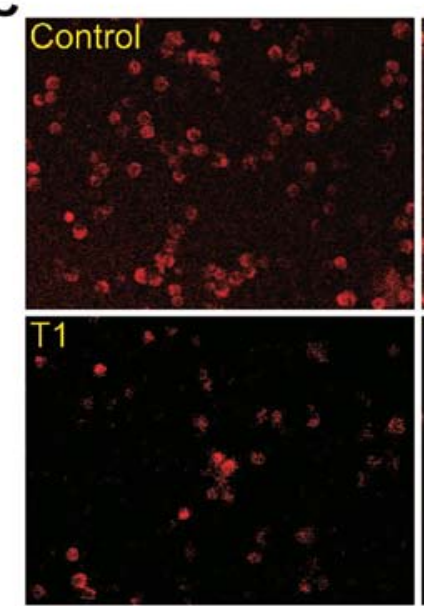

B

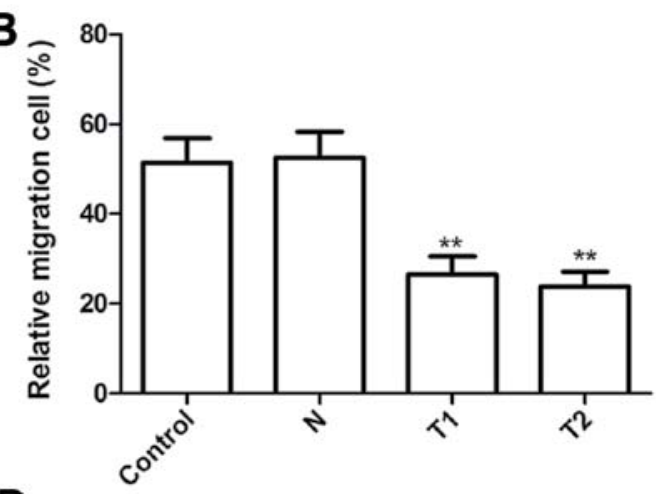

D
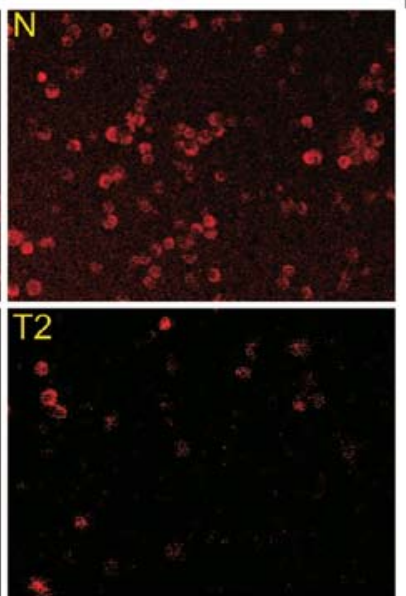

Figure 5. Knockdown of hTERT inhibits cancer cell migration and invasion in vitro. Cell migration (A and B) and cell invasion (C and D) of HeLa cells was determined; T1 and T2, hTERT siRNA clone; N, scramble siRNA clone; Control, parental cells; ${ }^{* *} \mathrm{P}<0.01 \mathrm{vs.} \mathrm{control.}$ 
A

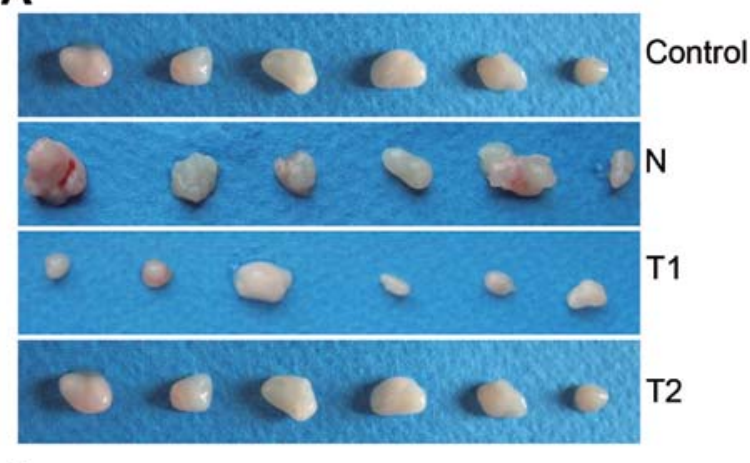

C

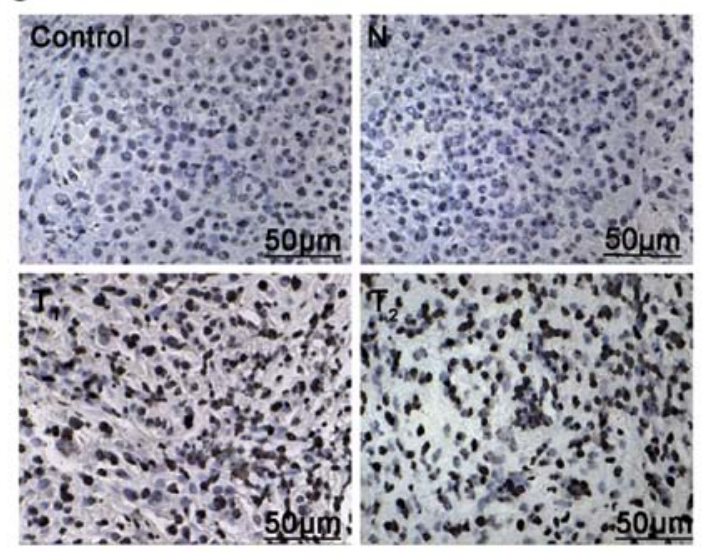

B

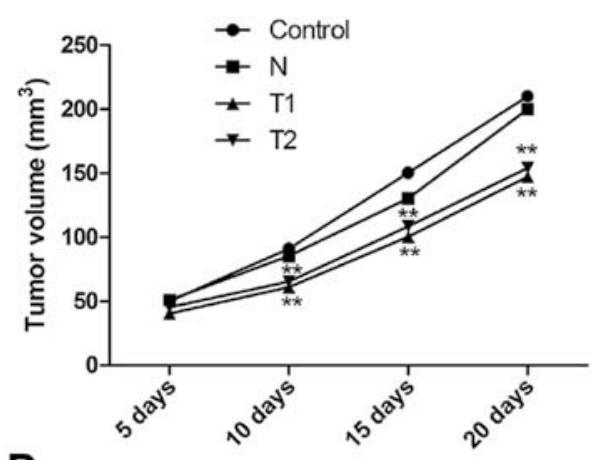

D

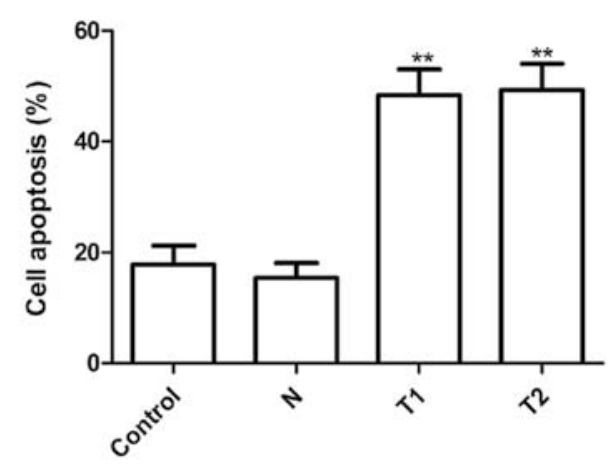

Figure 6. Knockdown of hTERT inhibits lung tumor growth in vivo. (A) Tumor weight of mice 21 days after hTERT siRNA; (B) Tumor volume of mice on days 5, 10, 15 and 20 after hTERT siRNA; (C) and (D) cell apoptosis was determined in vivo; Data are expressed as the means \pm SD; T1 and T2, hTERT siRNA clone; $\mathrm{N}$, scramble siRNA clone; Control, parental cells (untreated HeLa cells); $\mathrm{P}<0.05,{ }^{* *} \mathrm{P}<0.01$ vs. control.

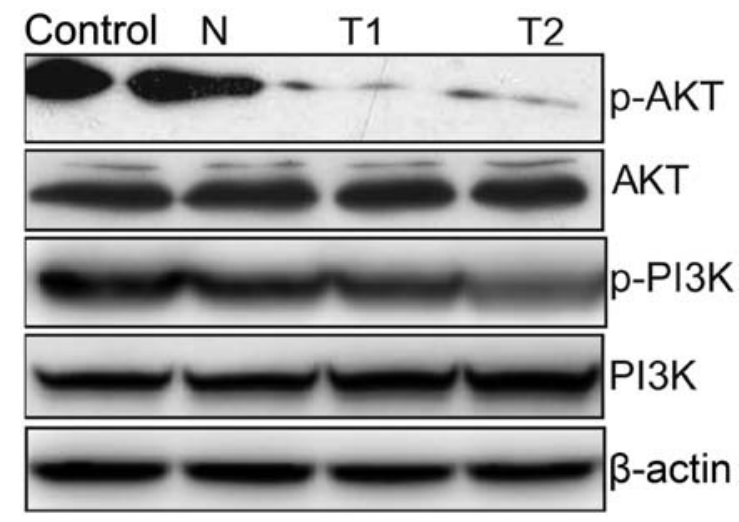

Figure 7. Knockdown of hTERT inhibits PI3K/AKT signaling in HeLa cells. Western blot analysis was performed using specific antibodies against the indicated proteins after treated with the indicated plasmid. Blots were reprobed for $\beta$-actin to normalize each lane for protein content; $\mathrm{T} 1$ and $\mathrm{T} 2$, hTERT siRNA clone; N, scramble siRNA clone; Control, parental cells.

results might indicate that knockdown of hTERT inhibits tumor cell growth, to some extent, by suppressing the PI3K/ AKT pathway.

\section{Discussion}

RNA interference (RNAi) has been proven to be a powerful tool for gene knockdown and holds great promise for the treat- ment of cancer (29). The key to the success of this method is to look for a gene which is expressed universally in cancer cells, but not in normal cells. hTERT appears to be such a candidate gene. Extensive studies showed that most normal human cells lack telomerase activity due to the stringent transcriptional repression of the hTERT gene, whereas the induction of hTERT expression and telomerase activation is in general a prerequisite step for malignant transformation of human cells (30-34). Hahn et al suggested that cancer cells undergo progressive telomere shortening, thereby triggering cellular senescence or apoptosis, and eventual loss of tumorigenic potential, if telomerase activity or hTERT expression is inhibited (35). Therefore, targeting telomerase or hTERT has been proposed as a novel anticancer strategy $(36,37)$. Extensive studies showed that silencing hTERT could inhibit cell proliferation and induce cell apoptosis in various cancer (15-20). Thus, targeted suppression of hTERT expression has potential for therapeutic strategy in cervical cancer. In the present study, we found that silencing of hTERT expression in HeLa cells by a specific shRNA results in decreased cell growth and increased cell apoptosis, which is consistent with previous reports (17,21-24).

Several reports have demonstrated that knockout of hTERT siRNA inhibits cell proliferation and telomerase activity in cervical cancer $(17,21-24)$. Natarajan et al found that stable suppression of hTERT expression led to significant slowing of the proliferative rates of cells lacking hTERT and to the attenuation of tumorigenic potential, and that suppression of hTERT by siRNA sensitized cancer cells to ionizing radiation 
and chemotherapeutic drugs known to induce DNA strand breaks (17). Kurvinen et al further showed that suppression of hTERT expression by siRNA inhibited telomerase activity and the length in human cervical cancer cells and that long-term suppression of telomerase expression by siRNA is an attainable goal, at least in a HeLa cell model system (23). Wang et al found that siRNA-hTERT effectively inhibited the hTERT expression, and induced apoptosis of HeLa cells via activating the mitochondrial signal transduction pathway (22). It was also reported that siRNA-hTERT induces apoptosis of HeLa cells (24). Recently, Zhang et al demonstrated that the silencing of hTERT could induce immediate growth arrest, enhance the $S$ phase in cell cycle study and lead to early apoptosis in human cervical cancer cells ( $\mathrm{SiHa}$ ), and that downregulation of hTERT enhanced radiosensitivity in SiHa cells (21). Although these studies showed that downregulation of hTERT by siRNA could inhibit cell proliferation, induced cell apoptosis, and enhanced radiation and chemotherapeutic drugs sensitivity for cervical cancer cell in vitro, whether silencing hTERT by siRNA affects cell migration and invasion of cervical cancer cells in vitro, and inhibit tumor growth in vivo was not reported. We investigated downregulation of hTERT by siRNA effect on cervical cancer cell growth in vitro and in vivo, and found that knockdown of hTERT by siRNA could significantly inhibit telomerase activity and length, suppress cell proliferation, cell migration and cell invasion, and induced cell apoptosis in vitro, and suppress tumor growth in vivo in cervical cancer. The in vivo tumor growth experiment clearly demonstrated impaired growth of the tumors formed by the hTERT siRNA transfected HeLa cells and induced apoptosis in pre-existing tumors. These studies imply that silencing hTERT might be an effective anticancer method for treatment of cervical cancer.

Many of the signal transduction pathways which have been shown to be involved in hTERT regulation are already well described in the context of the cellular DNA damage response, cell death induction or growth arrest in cancer cells (36). In this study, we mainly focused on the silencing of hTERT in PI3K/Akt signaling pathway because the PI3K/Akt signaling cascade is a central regulator for cell proliferation, growth and apoptosis (36). It has been shown that activated Akt in turn mediates the phosphorylation of hTERT, and enhances telomerase activity (38) due to hTERT protein with two putative Akt phosphorylation sites (39). Besides, hTERT phosphorylation by Akt and the active telomerase enzyme are thought to be necessary for its import into the nucleus $(40,41)$. In addition, hTERT has been found to make cancer cells more resistant against chemotherapeutic agents or radiation therapy via the PI3K/AKT pathway (42), therefore, we speculated that hTERT involved in tumor procession might be via PI3K/AKT pathway. We found that silencing of hTERT inhibited the tyrosine phosphorylation of AKT, and PI3K, which was in agreement with previous results (42), and indicate that knockdown of hTRET inhibits tumor cell growth, to some extent, by suppressing the PI3K/AKT pathway.

In conclusion, our results showed that knockdown of hTERT by siRNA could significantly inhibit telomerase activity and length, suppressed cell proliferation, cell cycle, cell migration and cell invasion, and induced cell apoptosis in vitro, and suppressed tumor growth in vivo in cervical cancer. These results suggest that silencing targeted hTERT may have therapeutic potential for treatment of cervical cancer.

\section{Acknowledgements}

This study was supported by Scientific Research Project of Jilin Provincial Bureau of Health (2013ZC005;2013Z028); Jilin Provincial Science and Technology Projects (20130101130JC); Norman Bethune Program of Jilin University (2012204); and The Project-sponsored by SRF for ROCS, SEM.

\section{References}

1. Richardson MA: Complementary and alternative therapy use in gynecologic oncology: implications for clinical practice. Gynecol Oncol 84: 360-362, 2002.

2. Richart RM: A modified terminology for cervical intraepithelial neoplasia. Obstet Gynecol 75: 131-133, 1990.

3. Jemal A, Siegel R, Ward E, Hao Y, Xu J and Thun MJ: Cancer statistics, 2009. CA Cancer J Clin 59: 225-249, 2009.

4. Jemal A, Bray F, Center MM, Ferlay J, Ward E and Forman D: Global cancer statistics. CA Cancer J Clin 61: 69-90, 2011.

5. Parkin DM, Bray F, Ferlay J and Pisani P: Global cancer statistics, 2002. CA Cancer J Clin 55: 74-108, 2005.

6. Toyoki H, Fujimoto J, Sato E, Sakaguchi H and Tamaya T: Clinical implications of expression of cyclooxygenase-2 related to angiogenesis in uterine endometrial cancers. Ann Oncol 16: $51-55,2005$.

7. Maher SG, Romero-Weaver AL, Scarzello AJ and Gamero AM: Interferon: cellular executioner or white knight? Curr Med Chem 14: 1279-1289, 2007

8. Shay JW and Wright WE: Role of telomeres and telomerase in cancer. Semin Cancer Biol 21: 349-353, 2011.

9. Kirkpatrick KL and Mokbel K: The significance of human telomerase reverse transcriptase (hTERT) in cancer. Eur J Surg Oncol 27: 754-760, 2001.

10. Cohen SB, Graham ME, Lovrecz GO, Bache N, Robinson PJ and Reddel RR: Protein composition of catalytically active human telomerase from immortal cells. Science 315: 18501853, 2007.

11. Carpentier C, Lejeune J, Gros F, et al: Association of telomerase gene hTERT polymorphism and malignant gliomas. J Neurooncol 84: 249-253, 2007.

12. Masutomi K and Hahn WC: Telomerase and tumorigenesis. Cancer Lett 194: 163-172, 2003.

13. Kyo S, Takakura M, Fujiwara T and Inoue M: Understanding and exploiting hTERT promoter regulation for diagnosis and treatment of human cancers. Cancer Sci 99: 1528-1538, 2008.

14. He X, Qiao Q, Ge N, Nan J, Shen S, Wang Z, Yang Y and Bao G: Irradiation-induced telomerase activity and gastric cancer risk: a case-control analysis in a Chinese Han population. BMC Cancer 10: 312-321, 2010.

15. Gandellini P, Folini M, Bandiera R, et al: Down-regulation of human telomerase reverse transcriptase through specific activation of RNAi pathway quickly results in cancer cell growth impairment. Biochem Pharmacol 73: 1703-1714, 2007.

16. Nakamura M, Masutomi K, Kyo S, et al: Efficient inhibition of human telomerase reverse transcriptase expression by RNA interference sensitizes cancer cells to ionizing radiation and chemotherapy. Hum Gene Ther 16: 859-868, 2005.

17. Natarajan S, Chen Z, Wancewicz EV, Monia BP and Corey DR: Telomerase reverse transcriptase (hTERT) mRNA and telomerase RNA (hTR) as targets for downregulation of telomerase activity. Oligonucleotides 14: 263-273, 2004.

18. de Souza Nascimento P, Alves G and Fiedler W: Telomerase inhibition by an siRNA directed against hTERT leads to telomere attrition in HT29 cells. Oncol Rep 16: 423-428, 2006.

19. Zhang PH, Zou L and Tu ZG: RNAi-hTERT inhibition hepatocellular carcinoma cell proliferation via decreasing telomerase activity. J Surg Res 131: 143-149, 2006.

20. Dong X, Liu A, Zer C, et al: siRNA inhibition of telomerase enhances the anti-cancer effect of doxorubicin in breast cancer cells. BMC Cancer 9: 133, 2009.

21. Zhang W and Xing L: RNAi gene therapy of SiHa cells via targeting human TERT induces growth inhibition and enhances radiosensitivity. Int J Oncol 43: 1228-1234, 2013. 
22. Wang R, Lin F, Wang X, et al: The therapeutic potential of survivin promoter-driven siRNA on suppressing tumor growth and enhancing radiosensitivity of human cervical carcinoma cells via downregulating hTERT gene expression. Cancer Biol Ther 6: 1295-1301, 2007.

23. Kurvinen K, Syrjanen S and Johansson B: Long-term suppression of telomerase expression in HeLa cell clones, transfected with an expression vector carrying siRNA targeting hTERT mRNA. Int J Oncol 29: 279-288, 2006.

24. Zhao Y, Ren JL, Zhang R, et al: Study on apoptosis of human cervical carcinoma HeLa cells with RNA interference targeting hTERT. Xi Bao Yu Fen Zi Mian Yi Xue Za Zhi 21: 524-526, 2005 (In Chinese).

25. Reynolds A, Leake D, Boese Q, Scaringe S, Marshall WS and Khvorova A: Rational siRNA design for RNA interference. Nat Biotechnol 22: 326-330, 2004.

26. Livak KJ and Schmittgen TD: Analysis of relative gene expression data using real-time quantitative PCR and the 2(-Delta Delta C(T)) method. Methods 25: 402-408, 2001.

27. Kim NW, Piatyszek MA, Prowse KR, et al: Specific association of human telomerase activity with immortal cells and cancer. Science 266: 2011-2015, 1994.

28. Lowry OH, Rosebrough NJ, Farr AL and Randall RJ: Protein measurement with the Folin phenol reagent. J Biol Chem 193 265-275, 1951.

29. Ramachandran PV and Ignacimuthu S: RNA interference as a plausible anticancer therapeutic tool. Asian Pac J Cancer Prev 13: 2445-2452, 2012.

30. Blackburn EH: Switching and signaling at the telomere. Cell 106: 661-673, 2001.

31. Boukamp P and Mirancea N: Telomeres rather than telomerase a key target for anti-cancer therapy? Exp Dermatol 16: 71-79, 2007.
32. Harley CB: Telomerase and cancer therapeutics. Nat Rev Cancer 8: 167-179, 2008.

33. Shay JW and Keith WN: Targeting telomerase for cancer therapeutics. Br J Cancer 98: 677-683, 2008.

34. Hahn WC, Counter CM, Lundberg AS, Beijersbergen RL, Brooks MW and Weinberg RA: Creation of human tumour cells with defined genetic elements. Nature 400: 464-468, 1999.

35. Hahn WC, Stewart SA, Brooks MW, et al: Inhibition of telomerase limits the growth of human cancer cells. Nat Med 5: 1164-1170, 1999.

36. Lamy E, Goetz V, Erlacher M,Herz C and Mersch-Sundermann V: hTERT: Another brick in the wall of cancer cells. Mutat Res 752: 119-128, 2013.

37. Chen H, Li Y and Tollefsbol TO: Strategies targeting telomerase inhibition. Mol Biotechnol 41: 194-199, 2009.

38. Cong YS, Wright WE and Shay JW: Human telomerase and its regulation. Microbiol Mol Biol Rev 66: 407-425, 2002.

39. Kang SS, Kwon T, Kwon DY and Do SI: Akt protein kinase enhances human telomerase activity through phosphorylation of telomerase reverse transcriptase subunit. J Biol Chem 274: 13085-13090, 1999.

40. Kimura A, Ohmichi M, Kawagoe J, et al: Induction of hTERT expression and phosphorylation by estrogen via Akt cascade in human ovarian cancer cell lines. Oncogene 23: 4505-4515, 2004.

41. Kawagoe J, Ohmichi M, Takahashi T, et al: Raloxifene inhibits estrogen-induced up-regulation of telomerase activity in a human breast cancer cell line. J Biol Chem 278: 43363-43372, 2003.

42. Ram R, Uziel O, Eldan O, et al: Ionizing radiation up-regulates telomerase activity in cancer cell lines by post-translational mechanism via ras/phosphatidylinositol 3-kinase/Akt pathway. Clin Cancer Res 15: 914-923, 2009. 